Groups Geom. Dyn. 4 (2010), 901-908

DOI $10.4171 / \mathrm{GGD} / 113$
Groups, Geometry, and Dynamics

(C) European Mathematical Society

\title{
Universal diagram groups with identical Poincaré series
}

\author{
Stephen J. Pride
}

\begin{abstract}
For a diagram group $G$, the first derived quotient $G_{1} / G_{2}$ is always free abelian (as proved by M. Sapir and V. Guba). However the second derived quotient $G_{2} / G_{3}$ may contain torsion. In fact, we show that for any finite or countably infinite direct product of cyclic groups $A$, there is a diagram group with second derived quotient $A$. We use that to construct families with the properties of the title.
\end{abstract}

Mathematics Subject Classification (2010). 20F05, 20F12, 20F65, 57M07, 20F14, 20J06, 57M05, 57M20.

Keywords. Diagram groups, derived quotient, FP-infinity, Poincaré series.

\section{Introduction}

A rewriting system $\mathcal{K}$ is a pair $\langle X ; \Phi\rangle$, where $X$ is a set (alphabet), and each $f \in \Phi$ is a pair of words on $X$, written as $f_{+1}=f_{-1}$. The elements of $\Phi$ are called rewriting rules. It is allowed that $f_{+1}$ and $f_{-1}$ are the same word. It is also allowed that for two rewriting rules $f, g$ the words $f_{+1}, g_{+1}$ are the same, and the words $f_{-1}, g_{-1}$ are the same. For example, the rewriting system $\left\langle x ; x^{2}=x, x=x, x=x\right\rangle$ has three rules.

Associated with $\mathcal{K}$ is a (combinational) 2-complex $\mathcal{C}(\mathcal{K})$. The underlying 1-skeleton is a graph in the sense of Serre [8]: the vertices are all the words on $X$ (i.e., $\left.X^{*}\right)$; the edges are triples of the form $\left(u, f_{+\varepsilon} \rightarrow f_{-\varepsilon}, v\right)\left(u, v \in X^{*}, f \in \Phi\right.$, $\varepsilon \pm 1)$; the inverse $e^{-1}$ of the edge $e=\left(u, f_{+\varepsilon} \rightarrow f_{-\varepsilon}, v\right)$ is $\left(u, f_{-\varepsilon} \rightarrow f_{+\varepsilon}, v\right)$. Note that if $f_{+1}$ and $f_{-1}$ are the same word, we will still regard $e^{+1}$ and $e^{-1}$ as different edges. The 2-cells are the quintuples $(u, f, v, g, w)\left(u, v, w \in X^{*}, f, g \in \Phi\right)$. The boundary of the 2-cell is the closed path $\left(u f_{+1} v, g, w\right)\left(u, f, v g_{-1} w\right)\left(u f_{-1} v, g, w\right)^{-1}$ $\left(u, f, v g_{+1} w\right)^{-1}$. The connected components of $\mathcal{C}(\mathcal{K})$ are in one-to-one correspondence with the elements of the semigroup defined by the rewriting system $\mathcal{K}$. The diagram group $\mathcal{D}(\mathcal{K}, v)$ of $\mathcal{K}$ is the fundamental group of the connected component of $\mathcal{C}(\mathcal{K})$ with $v$ as the basepoint.

These groups have been studied comprehensively by V. Guba and M. Sapir in the monograph [4], and the papers [6], [7]. Other interesting work about these groups 
can be found in [1], [2], [3], [5], [9]. There is a subclass of the class of diagram groups which is of interest, namely those which are universal. These groups have the property that they contain a copy of every countable diagram group (see [6], §5).

For any group $G$ we have the lower central series: $G_{1}=G$, and inductively, $G_{n+1}=\left[G_{n}, G\right]$. It is shown in [4], 111 , that for a diagram group $D, D_{1} / D_{2}$ is always free abelian. Since "diagram groups can be considered as 2-dimensional versions of free groups" [6], p. 2, line 11, it is reasonable to think that $D_{2} / D_{3}$, would also be free abelian. However, we will show that for any abelian group $A$ which is a finite or countably infinite direct product of cyclic groups, there is a diagram group $D$ such that $D_{2} / D_{3}$ is isomorphic to $A$ (Theorem 1).

For diagram groups arising from finite complete rewriting systems, the form of the canonical (minimal) presentation makes it easy to compute $D_{2} / D_{3}$. We will use this to exhibit, for any $k$, a family of universal diagram groups $\{D(i): i=1,2, \ldots, k\}$ which have very similar canonical presentations and the same integral homology, but which can be separated via their second derived quotients (Theorems 2, 3).

Throughout, $a^{b}$ will mean $b^{-1} a b$, and $[a, b]$ will mean $a^{-1} b^{-1} a b$.

For any elements $a, b \in G$ we write $\langle a, b\rangle$ for the element $[a, b] G_{3} \in G_{2} / G_{3}$. From the commutator calculus we have for any $a, b, c \in G$ :

(i) $\langle a, b c\rangle=\langle a, b\rangle\langle a, c\rangle$;

(ii) $\langle a, b\rangle=1$ if $a$ or $b$ is in $G_{2}$;

(iii) $\langle a, b\rangle=\langle b, a\rangle^{-1}$;

(iv) $\left\langle a^{-1} b a, c\right\rangle=\langle b, c\rangle=\left\langle b, a c a^{-1}\right\rangle$;

(v) $\left\langle a^{-1}, b\right\rangle=\langle a, b\rangle^{-1}=\left\langle a, b^{-1}\right\rangle$.

These formulae will be used without further mention.

We will make use of the method (and notation) found in [6], pp. 25-26, (see also [4], p. 53) for computing minimal presentations of diagram groups arising from complete rewriting systems. This requires the use of left and right forests of $\mathscr{C}(\mathcal{K})$. For our situation there will be a unique left (respectively right) forest consisting of the edges $\left(u, f_{+1} \rightarrow f_{-1}, v\right)^{ \pm 1}$, where $f \in F, f_{+1}$ and $f_{-1}$ are distinct words, and every proper prefix of $u f_{+1}$ (respectively every proper suffix of $\left.f_{+1} v\right)$ ) is irreducible (see [6], Lemma 6.3, Remark 6.4).

Acknowledgments. I thank Victor Guba, Mark Sapir, Bjorn Verduijn, and the anonymous referee. Thanks also to Steven Roper for the diagrams.

\section{The second derived quotients of some diagram groups}

Let $F$ be the free group with basis $X$, linearly ordered by $>$. The group $F_{2} / F_{3}$ is a free abelian group with free basis the elements $\left\langle x, x^{\prime}\right\rangle\left(x, x^{\prime} \in X, x>x^{\prime}\right)$.

Let $G=F / N$, where $N \triangleleft F_{2}$. Then $G_{2}=F_{2} / N$, and $G_{3}=F_{3} N / N$. Then

$$
G_{2} / G_{3}=\left(F_{2} / N\right) /\left(F_{3} N / N\right) \cong F_{2} /\left(F_{3} N\right) \cong\left(F_{2} / F_{3}\right) /\left(F_{3} N / F_{3}\right) .
$$


Suppose that $N$ is the normal closure of a set $R \subset F_{2}$. Then $F_{3} N / F_{3}$ is generated as a group by the elements $r F_{3}(r \in R)$. For $N$ is generated as a group by elements of the form $u^{-1} r u(u \in F, r \in R)$, so $F_{3} N / N$ is generated as a group by the elements $u^{-1} r u F_{3}=\left(r F_{3}\right)\left([r, u] F_{3}\right)=\left(r F_{3}\right)$. Thus if we can express each element $r F_{3}$ as an expression $\xi_{r}$ in terms of the basis elements $\left\langle x, x^{\prime}\right\rangle$, then $G_{2} / G_{3}$ is isomorphic to the free abelian group on the elements $\left\langle x, x^{\prime}\right\rangle\left(x, x^{\prime} \in X, x>x^{\prime}\right)$ factored by the subgroup generated by the elements $\xi_{r}(r \in R)$. Here is a couple of examples of how this works.

Example 1 (Generalised Thompson groups). Denote $\mathcal{P}=\mathcal{P}_{n}=\left\langle y ; y^{n}=y\right\rangle$ $(n>1)$. As shown in [4], pp. 56-57, the diagram group $G=\mathscr{D}(\mathcal{P}, y)$ has minimal presentation with generators $z_{0}, z_{1}, \ldots, z_{n-1}$, and defining relators as follows:

(i) $r_{i, k}=\left[z_{k}, z_{0}\right]^{-1}\left[z_{k}, z_{i}\right], 1 \leq i<k \leq n-1$;

(ii) $s_{i, k}=\left[z_{k}, z_{0}^{2}\right]^{-1}\left[z_{k}, z_{0} z_{i}\right], 1 \leq i, k \leq n-1, k-1 \leq i$;

(iii) $p=\left[z_{1}, z_{0}^{3}\right]^{-1}\left[z_{1}, z_{0}^{2} z_{n-1}\right]$.

Then in $F_{2} / F_{3}$ we have

(i') $\xi_{r_{i, k}}=\left\langle z_{k}, z_{0}\right\rangle^{-1}\left\langle z_{k}, z_{i}\right\rangle, 1 \leq i<k \leq n-1$;

(ii') $\xi_{s_{i, k}}=\left\langle z_{k}, z_{0}\right\rangle^{-2}\left\langle z_{k}, z_{0}\right\rangle\left\langle z_{k}, z_{i}\right\rangle=\left\langle z_{k}, z_{0}\right\rangle^{-1}\left\langle z_{k}, z_{i}\right\rangle, 1 \leq i, k \leq n-1$, $k-1 \leq i$

(iii') $\xi_{p}=\left\langle z_{1}, z_{0}\right\rangle^{-3}\left\langle z_{1}, z_{0}\right\rangle^{2}\left\langle z_{1}, z_{n-1}\right\rangle=\left\langle z_{1}, z_{0}\right\rangle^{-1}\left\langle z_{1}, z_{n-1}\right\rangle$.

Let $K$ be the subgroup generated by (i'), (ii'), (iii'). For $2 \leq k \leq n-1$ (and writing $={ }_{K}$ for equal modulo $K$ ) we have from $\left(i^{\prime}\right)$ :

$$
\left\langle z_{k}, z_{0}\right\rangle={ }_{K}\left\langle z_{k}, z_{i}\right\rangle \quad(1 \leq i<k) .
$$

Taking $i=k$ in (ii') we have

$$
\left\langle z_{k}, z_{0}\right\rangle={ }_{K}\left\langle z_{k}, z_{k}\right\rangle={ }_{K} 1 \quad(1 \leq i<k) .
$$

Thus, for $2 \leq k \leq n-1$, we have $\left\langle z_{k}, z_{i}\right\rangle={ }_{K} 1(0 \leq i<k \leq n-1)$. Then from (iii') we have $\left\langle z_{1}, z_{0}\right\rangle={ }_{K}\left\langle z_{n-1}, z_{1}\right\rangle^{-1}$, and since from above $\left\langle z_{n-1}, z_{1}\right\rangle={ }_{K} 1$, we obtain $\left\langle z_{1}, z_{0}\right\rangle={ }_{K}$ 1. Thus $G_{2} / G_{3}$ is trivial.

Example 2 (The -product of Guba/Sapir). Let $G, H$ be groups and let $Z$ be an infinite cyclic group generated by $z$. Then $G \cdot H$ is the free product $G * H * Z$ factored out by the normal closure of $\left\{\left[g^{z^{n}}, h\right]: g \in G, h \in H, n=0,1,2, \ldots\right\}$. This product is closed on the class of diagram groups [4], Theorem 8.6. The diagram group $\mathbb{Z} \bullet \mathbb{Z}$ is finitely generated but not finitely presented [4], Theorem 10.5. Let $G \cong F / N$ and $H \cong \widehat{F} / M$, where $F$ is free on $X, \widehat{F}$ is free on $Y$, and $M, N$ are the normal closures of sets $R \subseteq F_{2}, S \subseteq \hat{F}_{2}$, respectively. Then $P=G \bullet H$ has group presentation

$$
\left\langle X, Y, z ; R, S,\left[w^{z^{n}}, v\right](w \in F, v \in \widehat{F}, n=0,1,2, \ldots)\right\rangle .
$$


Thus $P_{2} / P_{3}$ is the free abelian group on the generators $\left\langle x, x^{\prime}\right\rangle\left(x, x^{\prime} \in X, x>x^{\prime}\right)$, $\left\langle y, y^{\prime}\right\rangle\left(y, y^{\prime} \in Y, y>y^{\prime}\right),\langle x, y\rangle(x \in X, y \in Y),\langle x, z\rangle(x \in X),\langle y, z\rangle$ $(y \in Y)$, with relators $\xi_{r}(r \in R), \xi_{s}(s \in S), \xi_{\left[w^{\left.z^{n}, v\right]}\right.}\left(=\xi_{[w, v]}\right)(w \in F, v \in \hat{F}$, $n=0,1,2, \ldots)$. Thus

$$
P_{2} / P_{3} \cong G_{2} / G_{3} \times H_{2} / H_{3} \times A(X) \times A(Y),
$$

where $A(X), A(Y)$ are the free abelian groups on the generating sets $\{\langle x, z\rangle: x \in X\}$, $\{\langle y, z\rangle: y \in Y\}$, respectively.

Theorem 1. For any abelian group $A$ which is a finite or countably infinite direct product of cyclic groups, there is a diagram group $G$ such that $G_{2} / G_{3} \cong A$.

Proof. Consider the complete rewriting system

$$
\begin{gathered}
\mathcal{K}=\mathcal{K}_{n, m}=\langle a, y, c ; h, f, g\rangle, \\
h: a y=a, \quad f: y^{n}=y^{m}, \quad g: y c=c,
\end{gathered}
$$

$0<m \leq n$. The generators of the groups $G(n, m)=\mathscr{D}(\mathcal{K}, a c)$ are $z=(a, f, c)$ and $t=(a, g, 1)$, and the defining relators $r_{i}$ are coded by the quintuples $\left(a, f, y^{i}, f, c\right)$, $0 \leq i<n$. Then according to [6], p. 25, $r_{i}$ is the word

$$
\left[a, f, y^{i+n} c\right]^{-1}\left[a, f, y^{i+m} c\right]^{[a, f, c]} .
$$

Now the negative edge from the right tree that is assigned to $y^{p} c$ is $\left(y^{p-1}, g, 1\right)$. Thus

$$
\left[a, f, y^{p} c\right]=\left[a, f, y^{p-1} c\right]^{[a, g, 1]},
$$

and so by induction $\left[a, f, y^{p} c\right]$ is the word $z^{t^{p}}$. Hence $r_{i}$ is the word

$$
t^{-(i+n)} z^{-1} t^{i+n} z^{-1} t^{-(i+m)} z t^{(i+m)} z \text {. }
$$

Now $r_{i}$ is freely equivalent to $\left[t^{i+n}, z\right] z^{-1}\left[z, t^{i+m}\right] z$ and thus freely equivalent to $\left[z, t^{i+n}\right]^{-1}\left[z, t^{i+m}\right]\left[\left[z, t^{i+m}\right], z\right]$. It thus follows that $r_{i} F_{3}=\langle z, t\rangle^{m-n}$, so $G(n, m)_{2} / G(n, m)_{3}$ is cyclic of order $n-m$ (infinite cyclic if $n-m=0$ ).

Now the class of diagram groups is closed under countable direct products [7], Theorem 2.5. Also, if $G=\prod_{j \in J} G(j)$, then $G_{2} / G_{3} \cong \prod_{j \in J} G(j)_{2} / G(j)_{3}$. So using the groups above we obtain our result.

\section{Some universal diagram groups}

In this section we will need to regard rewriting systems as directed 2-complexes with one vertex (see [6], §1 and Theorem 4.3). Also, the material on morphisms of directed 2-complexes, [6], p. 17, and the concept of a universal directed 2-complex, [6], p. 18, will be needed. 
In [6], Theorem 5.5, it was shown that $\mathscr{H}=\left\langle x ; x=x, x^{2}=x\right\rangle$ is a universal directed 2-complex. The authors also showed that there is a non-singular map from $\mathscr{H}$ into $\mathcal{V}_{3,2}=\left\langle y ; y^{3}=y^{2}\right\rangle$, and thus $\mathcal{V}_{3,2}$ is universal [6], Theorem 5.6. The proof can be generalised:

Theorem 2. $\mathcal{V}_{n, m}=\left\langle y ; y^{n}=y^{m}\right\rangle(n>m>1)$ is universal for $\frac{3 n}{5}<m \leq \frac{5 n}{7}$.

Proof. Consider the map

$$
\phi: \mathscr{H} \rightarrow \mathcal{V}=\mathcal{V}_{n, m},
$$

where the edge $x$ is sent to the path $y^{4(n-m)}$, and the two 2-cells $f: x=x$ and $g: x^{2}=x$ are sent respectively to the 2-paths $A, B$ in Diagram 1 in Figure 1 (where a directed line labelled by a positive integer $k$ is shorthand for the directed path $y^{k}$, and where $\varepsilon=0$ if $m$ is even, and $\varepsilon=1$ if $m$ is odd). The proof is to check that for any reduced diagram $\Delta$ over $\mathscr{H}$, when we fill in each $f$-cell by a copy of $A$, and fill in each $g$-cell with a copy of $B$, no dipole can arise.

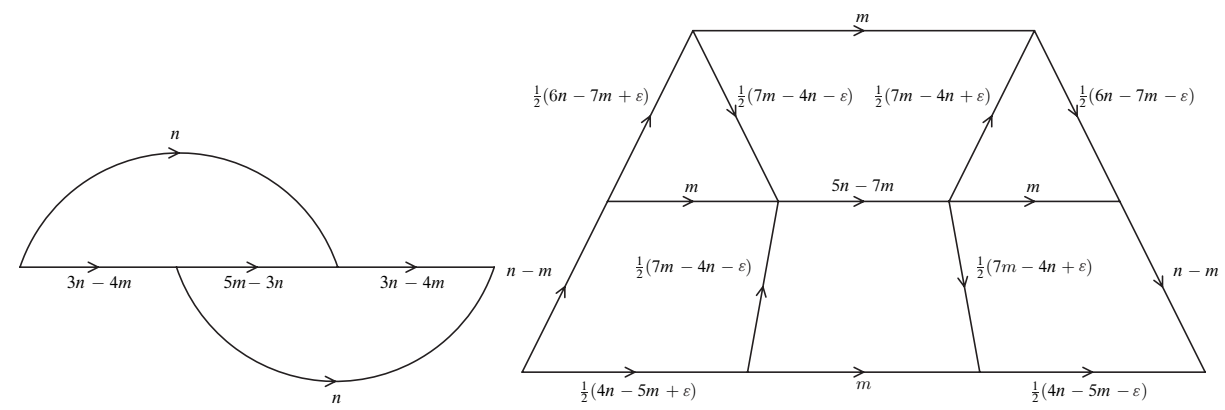

Figure 1. Diagram 1

(1) The only interaction of two $f 2$-cells in a reduced $\mathscr{H}$ diagram is if the two copies of $f$ are both positive, or both are negative. Assuming they are both positive (the negative case is similar), then when these cells are filled in by copies of $A$, the potential pair (shaded) is not a dipole (see the diagram in Figure 2).

(2) The possible interactions of two $g$ 2-cells in a reduced diagram over $\mathscr{H}$ are as in Diagram 3 in Figure 3 (up to rotation by $\pi$ around the central line; or, by reflection in a mirror perpendicular to the central line and the arrows reversed). When these cells are filled in by the appropriate copies of $B$, the only potential pairs for dipoles (shaded in the diagram) cannot be such.

(3) Clearly, no dipole can arise from an interaction between an $A$ diagram and a $B$ diagram. 

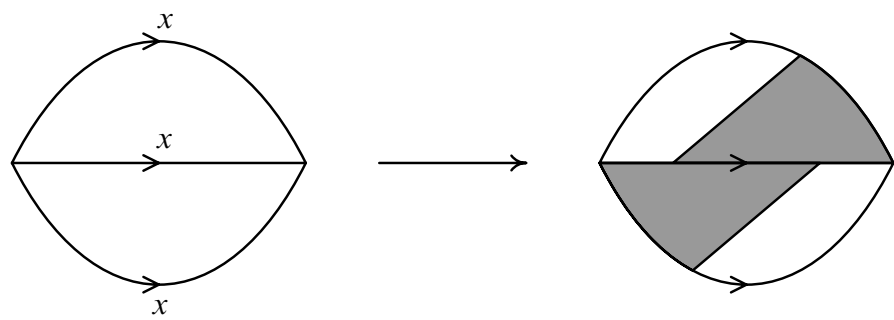

Figure 2. Diagram 2
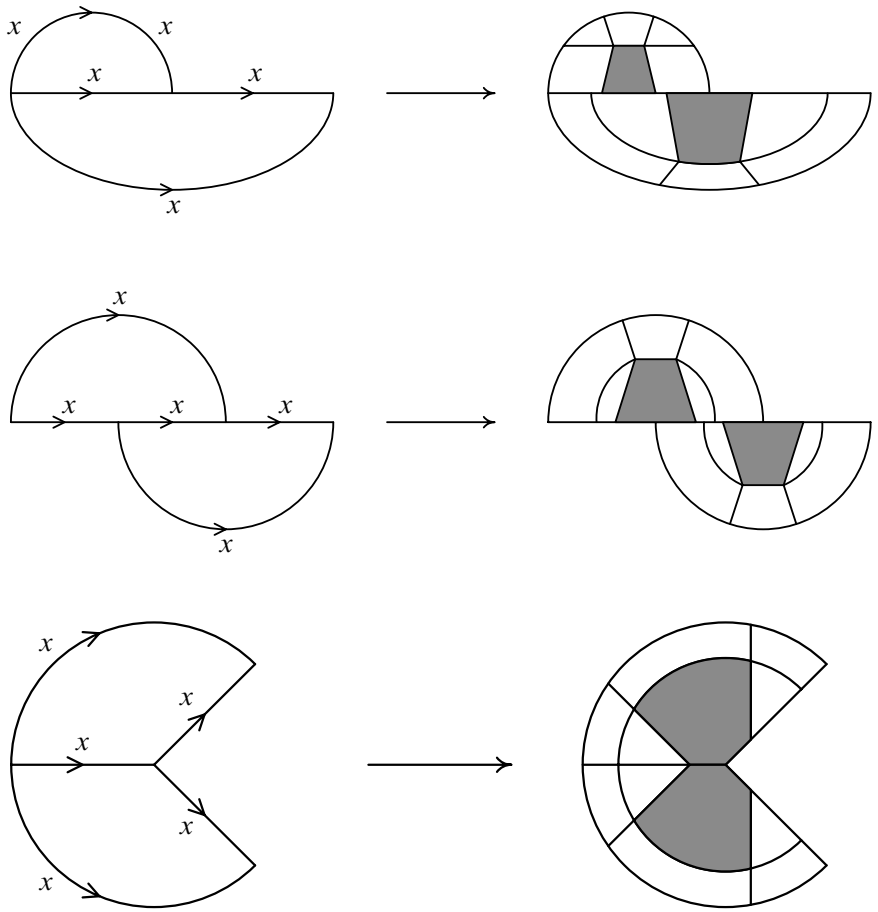

Figure 3. Diagram 3

Theorem 3. For a fixed $n$ there is a family of diagram groups $G(n, m)\left(\frac{3 n}{5}<m \leq \frac{5 n}{7}\right)$ with the following properties:

(i) The groups are universal.

(ii) Their minimal presentations each have the same number of generators, and each have the same number of defining relators.

(iii) The groups are all of type $\mathrm{FP}_{\infty}$, and all have the same Poincaré series.

However, no two groups in the family are isomorphic. 
Proof. By [6], Lemma 5.1 (2), the inclusion

$$
\varphi: \mathcal{V}_{n, m} \rightarrow \mathcal{K}_{n, m}
$$

is non-singular. Thus we have the induced group homomorphism

$$
\varphi_{*}: \mathscr{D}\left(\mathcal{V}_{n, m}, y^{4(n-m)}\right) \rightarrow \mathscr{D}\left(\mathcal{K}_{n, m}, y^{4(n-m)}\right),
$$

which is injective. Then, by [6], Corollary 3.6,

$$
\mathscr{D}\left(\mathcal{K}_{n, m}, a\right) \times \mathscr{D}\left(\mathcal{K}_{n, m}, y^{4(n-m)}\right) \times \mathscr{D}\left(\mathcal{K}_{n, m}, c\right)
$$

is imbedded into $\mathscr{D}\left(\mathcal{K}_{n, m}, a y^{4(n-m)} c\right)$. By [6], Corollary 3.5, $\mathscr{D}\left(\mathcal{K}_{n, m}, a y^{4(n-m)} c\right)$ is isomorphic to $\mathscr{D}\left(\mathcal{K}_{n, m}, a c\right)$. Thus $G(n, m)=\mathscr{D}\left(\mathcal{K}_{n, m}, a c\right)$ is universal.

Note that for a fixed $n$, the minimal presentations of the groups $\mathscr{D}\left(\mathcal{K}_{n, m}, a c\right)$ $(1 \leq m<n)$ all have the same number of generators (namely 2$)$ and the same number of relators (namely $n$ ). See Theorem 1 .

Also, for the minimal free resolution (see [6], Theorem 9.2)

$$
0 \leftarrow \mathbb{Z} \leftarrow F_{0} \leftarrow F_{1} \leftarrow F_{2} \leftarrow \cdots \leftarrow F_{p} \leftarrow \cdots
$$

the basis of $F_{p}$ is:

(i) $(a c)$ if $p=0$;

(ii) $(a, f, c),(a, g, 1)$ if $p=1$;

(iii) $\left(a, f, y^{i}, f, c\right)(1 \leq i<n)$ and $\left(a, f, y^{n-1}, g, 1\right)$ if $p=2$;

and

(iv) for $p \geq 3$ the basis is:

$$
\left(a, f, y^{i_{1}}, f, y^{i_{2}}, \ldots, y^{i_{p-1}}, f, c\right), \quad 1 \leq i_{k} \leq n-1,1 \leq k \leq p-1,
$$

and

$$
\left(a, f, y^{i_{1}}, f, y^{i_{2}}, \ldots, y^{i_{p-2}}, f, y^{n-1}, g, 1\right), \quad 1 \leq i_{k} \leq n-1,1 \leq k \leq p-2 .
$$

Thus the groups have the same Poincaré series, namely

$$
\begin{aligned}
P(t) & =1+2 t+n t^{2}\left(1+(n-1) t+((n-1) t)^{2}+((n-1) t)^{3}+\cdots\right) \\
& =1+2 t+\frac{n t^{2}}{1-(n-1) t} .
\end{aligned}
$$

However, as shown in $\S 2, G(n, m)_{2} / G(n, m)_{3} \cong \mathbb{Z}_{n-m}(1 \leq m \leq n)$, so for a fixed $n$, the groups $G(n, m)(1 \leq m \leq n)$ are distinct. 


\section{References}

[1] G. N. Arzhantseva, V. S. Guba, and M. V. Sapir, Metrics on diagram groups and uniform embeddings in a Hilbert space. Comment. Math. Helv. 81 (2006), 911-929.Zbl 1166.20031 MR 2271228

[2] D. S. Farley, Finiteness and CAT(0) properties of diagram groups. Topology 42 (2003), 1065-1082. Zbl 1044.20023 MR 1978047

[3] D. S. Farley, Homological and finiteness properties of picture groups. Trans. Amer. Math. Soc. 357 (2005), 3567-3584. Zbl 1137.20032 MR 2146639

[4] V. S. Guba and M. V. Sapir, Diagram groups. Mem. Amer. Math. Soc. 130 (1997), no. 620. Zbl 0930.20033 MR 1396957

[5] V. S. Guba and M. V. Sapir, Rigidity properties of diagram groups. Internat. J. Algebra Comput. 12 (2002), 9-17. Zbl 1031.20037 MR 1902358

[6] V. S. Guba and M. V. Sapir, Diagram groups and directed 2-complexes: homotopy and homology. J. Pure Appl. Algebra 205 (2006), 1-47. Zbl 1163.20027 MR 2193190

[7] V. S. Guba and M. V. Sapir, Diagram groups are totally orderable. J. Pure Appl. Algebra 205 (2006), 48-73. Zbl 1093.20023 MR 2193191

[8] J.-P. Serre, Trees. Springer-Verlag, Berlin 1980. Zbl 0548.20018 MR 0607504

[9] B. Wiest, Diagram groups, braid groups, and orderability. J. Knot Theory Ramifications 12 (2003), 321-332. Zbl 1089.20023 MR 1983088

Received October 19, 2009; revised June 15, 2010

S. J. Pride, Department of Mathematics, University of Glasgow, University Gardens, Glasgow G12 8QW, UK

E-mail: Stephen.Pride@glasgow.ac.uk 